Proceedings of the Edinburgh Mathematical Society (2004) 47, 695-707 (C)

DOI:10.1017/S001309150200072X Printed in the United Kingdom

\title{
REPRESENTATION OF ATOMIC OPERATORS AND EXTENSION PROBLEMS
}

\author{
EUGENE STEPANOV \\ Dipartimento di Matematica, Universitá di Pisa, via Buonarroti 2, \\ 56127 Pisa, Italy (e.stepanov@sns.it)
}

(Received 25 July 2002)

\begin{abstract}
The notion of an atomic operator between spaces of measurable functions was introduced in 2002 in a paper by Drakhlin, Ponosov and Stepanov in order to provide a reasonable generalization of local operators useful for applications. It has been shown that, roughly speaking, atomic operators amount to compositions of local operators with shifts. A natural problem is then when a continuousin-measure atomic operator can be represented as a composition of a Nemytskii (composition) operator generated by a Carathéodory function, and a shift operator. In this paper we will show that the answer to this question is inherently related to the possibility of extending an atomic operator with continuity from a space of functions measurable with respect to some $\sigma$-algebra to a larger space of functions measurable with respect to a larger $\sigma$-algebra, as well as to the possibility of extending any $\sigma$-homomorphism from a smaller-measure algebra to a $\sigma$-homomorphism on a larger-measure algebra. We characterize precisely the condition on the respective $\sigma$-algebras which provides such possibilities and induces the positive answer to the above representation problem.
\end{abstract}

Keywords: local operators; atomic operators; $\sigma$-homomorphism; extension

2000 Mathematics subject classification: Primary 47B38; 47A67; 34K05

\section{Introduction}

In [3] a particular class of operators, called atomic operators and generalizing the notion of a local operator between ideal function spaces, was introduced and many of its analytic properties - like acting, boundedness, continuity, compactness and convergence in various topologies - were studied. We recall here that this class contains local (in particular, Nemytskiî) operators, shift operators and is closed with respect to compositions. This study was to a large extent inspired by stochastic applications. For instance, atomic operators arise naturally in the problem of finding periodic (in distribution) solutions to stochastic differential equations. Moreover, many problems for stochastic dynamical systems can be reduced to the study of atomic operators.

The representation theorem proved in $[\mathbf{3}]$ asserts that, roughly speaking, an atomic operator is always a composition of a local operator and a shift. However, even if the atomic operator is continuous, the respective local operator is not necessarily a Nemytskiĩ operator generated by some Carathéodory function, since in general it will be acting between the spaces of functions measurable with respect to different $\sigma$-algebras. In fact, the same paper provides examples of continuous atomic operators arising in stochastic 
applications that cannot be represented as a composition of a Nemytskiǐ operator generated by a Carathéodory function, and a shift. Nevertheless, the question of when such a representation exists is of utmost importance for applications, for instance, for the purpose of seeking invariant measures or fixed points (maybe appropriately generalized) of atomic operators.

The above representation problem is inherently related to another two problems. Namely, suppose that $\left(\Omega, \Sigma^{\prime}, \mu\right)$ is a measure space and that $\Sigma \subset \Sigma^{\prime}$ is some smaller $\sigma$-algebra. The first problem is when an atomic operator defined over a space of functions measurable with respect to $\Sigma$ can be extended with continuity to the bigger space of functions measurable with respect to $\Sigma^{\prime}$. The second problem is when a nullset-preserving $\sigma$-homomorphism between $\Sigma$ and any other $\sigma$-algebra of sets can be extended to the whole $\Sigma^{\prime}$. A related question is when such a $\sigma$-homomorphism can be represented by a point map [8]. In this paper we show that all the above problems are in fact equivalent and admit a positive answer if and only if $\Sigma$ satisfies the so-called $\Omega$-condition, which was recently introduced in [7]. Moreover, for standard measure spaces we are able to completely characterize the $\Omega$-condition on $\sigma$-subalgebras in terms of the existence of a piecewise-injective generating function.

\section{Notation and preliminaries}

Let $\left(\Omega_{1}, \Sigma_{1}, \mu_{1}\right)$ and $\left(\Omega_{2}, \Sigma_{2}, \mu_{2}\right)$ be two measure spaces, and let $\Sigma_{1}^{0} \subset \Sigma_{1}, \Sigma_{2}^{0} \subset \Sigma_{2}$ be the $\sigma$-ideals of the $\mu_{1}$ - and $\mu_{2}$-nullsets, respectively. We denote by $\tilde{\Sigma}_{i}:=\Sigma_{i} / \Sigma_{i}^{0}$, $i=1,2$, the respective measure algebras (see $\S 42$ of $[8]$ ). The elements of $\tilde{\Sigma}_{i}$ (i.e. the equivalence classes of sets) will be denoted $\tilde{e}_{i}$ or $\left[e_{i}\right], i=1,2$. Further on we will, however, frequently abuse the notation and identify the elements of the measure algebras $\tilde{\Sigma}_{i}$ with the elements of the respective original $\sigma$-algebras of sets $\Sigma_{i}$. A map $F: \tilde{\Sigma}_{1} \rightarrow \tilde{\Sigma}_{2}$ is called a $\sigma$-homomorphism, if $F\left(\Omega_{1}\right)=\Omega_{2}, F\left(\Omega_{1} \backslash e\right)=\Omega_{2} \backslash F(e)$ whenever $e \in \tilde{\Sigma}_{1}$ and

$$
F\left(\bigsqcup_{i=1}^{\infty} e_{i}\right)=\bigsqcup_{i=1}^{\infty} F\left(e_{i}\right),
$$

for any pairwise disjoint collection of $\left\{e_{i}\right\}_{i=1}^{\infty} \subset \tilde{\Sigma}_{1}$, where $\bigsqcup$ stands for the disjoint union. Every $\left(\Sigma_{2}, \Sigma_{1}\right)$-measurable map $g: \Omega_{2} \rightarrow \Omega_{1}$ satisfying

$$
\mu_{2}\left(g^{-1}\left(e_{1}\right)\right)=0 \quad \text { when } \mu_{1}\left(e_{1}\right)=0
$$

generates a $\sigma$-homomorphism according to the formula $F\left(\tilde{e}_{1}\right):=\left[g^{-1}\left(e_{1}\right)\right]$. The latter $\sigma$-homomorphism is said to be induced by a point map $g$, and in this case we will write $F=g^{-1}$.

A measure space $(\Omega, \Sigma, \mu)$ is called standard if $\Omega$ is a Polish space and $\Sigma$ is either the Borel $\sigma$-algebra or its completion with respect to finite Borel measure $\mu$. Below all the measure spaces we will be dealing with are assumed to be complete and all the measures are assumed to be finite.

By $L^{0}(\Omega, \Sigma, \mu ; \mathcal{X})$, where $\mathcal{X}$ is a separable metric space, we denote the space of (classes of) $\mathcal{X}$-valued measurable functions equipped with the usual metric inducing the topology 
of convergence in measure. Whenever there is no possibility of confusion, the references to $\mathcal{X}, \Omega, \Sigma$ and/or $\mu$ will be omitted.

\section{Extension of atomic operators}

For the sake of brevity denote $X_{i}:=L^{0}\left(\Omega_{i}, \Sigma_{i} ; \mathcal{X}_{i}\right), i=1,2$. Recall the following notion introduced in [3].

Definition 3.1. An operator $T: X_{1} \rightarrow X_{2}$ is called atomic with respect to the $\sigma$-homomorphism $F: \tilde{\Sigma}_{1} \rightarrow \tilde{\Sigma}_{2}$ if $\left.T(x)\right|_{F\left(e_{1}\right)}=\left.T(y)\right|_{F\left(e_{1}\right)}$ whenever $\left.x\right|_{e_{1}}=\left.y\right|_{e_{1}}$ for $x, y \in X_{1}$.

We will further omit the reference to the particular $\sigma$-homomorphism if it is unnecessary.

We remark that in $[\mathbf{3}]$ a slightly different definition of an atomic operator was given, namely, the operator $T: X_{1} \rightarrow X_{2}$ was called atomic with respect to the nullset-preserving $\sigma$-homomorphism between the original $\sigma$-algebras $F: \Sigma_{1} \rightarrow \Sigma_{2}$ if $\left.T(x)\right|_{F\left(e_{1}\right)}=\left.T(y)\right|_{F\left(e_{1}\right)}$ whenever $\left.x\right|_{e_{1}}=\left.y\right|_{e_{1}}$ for $x, y \in X_{1}$. The requirement that $F$ be nullset preserving means that $\mu_{2}\left(F\left(e_{1}\right)\right)=0$ whenever $\mu_{1}\left(e_{1}\right)=0$. Clearly, every nullsetpreserving $\sigma$-homomorphism $F: \Sigma_{1} \rightarrow \Sigma_{2}$ between the original $\sigma$-algebras generates a $\sigma$-homomorphism $\tilde{F}$ of the respective measure algebras according to the formula $\tilde{F}\left(\tilde{e}_{1}\right):=\left[F\left(e_{1}\right)\right]$. Vice versa, given a $\sigma$-homomorphism between the measure algebras $\tilde{F}: \tilde{\Sigma}_{1} \rightarrow \tilde{\Sigma}_{2}$, provided that the measure space $\left(\Omega_{2}, \Sigma_{2}, \mu_{2}\right)$ is complete, we can construct a nullset-preserving $\sigma$-homomorphism $F: \Sigma_{1} \rightarrow \Sigma_{2}$ between the original $\sigma$ algebras satisfying $\left[F\left(e_{1}\right)\right]:=\tilde{F}\left(\tilde{e}_{1}\right)$. The respective construction is provided by the formula $F\left(e_{1}\right):=\pi\left(\tilde{F}\left(\tilde{e}_{1}\right)\right)$, where $\pi: \tilde{\Sigma}_{2} \rightarrow \Sigma_{2}$ is the lifting map satisfying $\left[\pi\left(\tilde{e}_{2}\right)\right]=\tilde{e}_{2}$. The existence of the latter is ensured by the von Neumann-Maharam lifting theorem (Theorem 4.4 in [4]), for which completeness of $\left(\Omega_{2}, \Sigma_{2}, \mu_{2}\right)$ is essential. Therefore, the definition of atomic operator given in $[\mathbf{3}]$ is equivalent to the above one provided one works with complete measure spaces.

The notion of an atomic operator seems to be just a reasonable generalization of the classical notion of a local operator for many applications. In fact, a local operator between spaces of measurable functions is just an atomic one with respect to the identity $\sigma$-homomorphism. In particular, a Nemytskiǐ operator $N_{f}: L^{0}\left(\Omega ; \mathcal{X}_{1}\right) \rightarrow L^{0}\left(\Omega ; \mathcal{X}_{2}\right)$ defined by the formula $\left(N_{f} u\right)(\omega):=f(\omega, u(\omega))$ for a given function $f: \Omega \times \mathcal{X}_{1} \rightarrow \mathcal{X}_{2}$ is local, and hence, atomic. But the class of atomic operators is, in fact, much wider and also embodies a lot of operators which though being non-local have many properties similar to those of local operators. Here we enlist some of the most important examples of atomic operators.

Example 3.2. The examples below were pointed out in $[\mathbf{3}]$.

(1) Let $g: \Omega_{2} \rightarrow \Omega_{1}$ be a $\left(\Sigma_{2}, \Sigma_{1}\right)$-measurable function satisfying (2.1). Then the shift operator $T_{g}: X_{1} \rightarrow X_{2}$ defined by the formula

$$
\left(T_{g} u\right)\left(\omega_{2}\right):=u\left(g\left(\omega_{2}\right)\right) \text { for } \mu_{2} \text {-a.e. } \omega_{2} \in \Omega_{2}
$$

is atomic with respect to the $\sigma$-homomorphism induced by $g$. 
(2) A composition of two atomic operators is still atomic. In particular, a composition of a local (e.g. Nemytskiǐ) operator and a shift is atomic, though, generally speaking, not local.

(3) Every linear continuous-in-measure disjointness preserving $(D-)$ operator (i.e. an operator sending functions with disjoint supports into functions with disjoint supports) is atomic. Note that nonlinear $D$-operators in general need not be atomic.

(4) Atomic operators arise naturally in stochastic applications. For instance, a problem of finding a strong periodic solution to a stochastic differential equation can be naturally reduced to a fixed-point problem for an operator which is a composition of the so-called Wiener shift and the evolution (propagation) operator along the trajectories of the stochastic differential equation. Since the latter operator is known to be local, the original problem is reduced to finding fixed points of an atomic operator.

We now consider the following problems, which are quite closely related to each other.

Problem 3.3. In $[\mathbf{3}]$ it has been shown that every atomic operator $T$ can in fact be represented as a composition $T=N \circ T_{F}$ of a local operator and some generalized shift operator $T_{F}$ with respect to a $\sigma$-homomorphism $F$. Suppose that the atomic operator $T: X_{1} \rightarrow X_{2}$ is continuous in measure. We would like to know when the local operator $N$ involved in the respective representation is a Nemytskiĩ operator generated by a Carathéodory function.

Problem 3.4. Let $\Sigma_{1} \subset \Sigma_{1}^{\prime}$, where $\Sigma_{1}^{\prime}$ is some larger $\sigma$-algebra of subsets of $\Omega$. We would like to know under which condition every continuous-in-measure atomic operator $T: X_{1} \rightarrow X_{2}$ can be extended to a continuous-in-measure atomic operator $T^{\prime}: X_{1}^{\prime} \rightarrow X_{2}$ over the larger space $X_{1}^{\prime}:=L^{0}\left(\Omega_{1}, \Sigma_{1}^{\prime} ; \mathcal{X}_{1}\right)$.

Problem 3.5. Let $F: \tilde{\Sigma}_{1} \rightarrow \tilde{\Sigma}_{2}$ be a $\sigma$-homomorphism. We would like to know when it can be extended to a $\sigma$-homomorphism $F^{\prime}: \tilde{\Sigma}_{1}^{\prime} \rightarrow \tilde{\Sigma}_{2}$.

The answers to the above problems we provide here involve the following notion which first appeared in [7].

Definition 3.6. Let $\Sigma_{1} \subset \Sigma_{1}^{\prime}$ be $\sigma$-algebras of subsets of $\Omega_{1}$. Then $\Sigma_{1}$ is said to satisfy the $\Omega$-condition with respect to $\Sigma_{1}^{\prime}$ (written $\Sigma_{1} \in \Omega\left(\Sigma_{1}^{\prime}\right)$ ) if there is an at-mostcountable cover of $\Omega_{1}$ by pairwise disjoint sets $\Omega_{1}=\bigsqcup_{j} \Omega_{1}^{j}, \Omega_{1}^{j} \in \Sigma_{1}^{\prime}$, such that for each $j \in \mathbb{N}$ one has $\Sigma_{1} \cap \Omega_{1}^{j}=\Sigma_{1}^{\prime} \cap \Omega_{1}^{j}$.

It is worth remarking that the same notion of $\Omega$-condition can be extended in an obvious way to measure algebras, namely, by saying that $\tilde{\Sigma}_{1} \in \Omega\left(\tilde{\Sigma}_{1}^{\prime}\right)$ if $\Sigma_{1} \cap \Omega_{1}^{j} / \Sigma_{1}^{0}=$ $\Sigma_{1}^{\prime} \cap \Omega_{1}^{j} / \Sigma_{1}^{0}$ for each $j \in \mathbb{N}$ and for some partition of $\Omega_{1}$ by an at-most-countable number of disjoint sets $\left\{\Omega_{1}^{j}\right\}_{j \in \mathbb{N}}$, where $\Sigma_{1}^{0}$ stands for the $\sigma$-ideal of $\mu_{1}$-nullsets.

The following result gives the answer to Problem 3.4. Note that its proof also uses the answer to Problem 3.5 given by Proposition 5.1. 
Theorem 3.7. Let $\tilde{\Sigma}_{1} \in \Omega\left(\tilde{\Sigma}_{1}^{\prime}\right)$. Then every atomic operator $T: X_{1} \rightarrow X_{2}$ admits a (not necessarily unique) extension to an atomic operator $T^{\prime}: X_{1}^{\prime} \rightarrow X_{2}$. Moreover, $T^{\prime}$ is continuous in measure whenever $T$ is continuous in measure.

Proof. Let $T: X_{1} \rightarrow X_{2}$ be atomic with respect to the $\sigma$-homomorphism $F$ : $\tilde{\Sigma}_{1} \rightarrow \tilde{\Sigma}_{2}$. According to Proposition 5.1 proven below, the latter admits an extension to $F^{\prime}: \tilde{\Sigma}_{1}^{\prime} \rightarrow \tilde{\Sigma}_{2}$. We now use Lemma 3.8 below to find the desired extension $T^{\prime}$ of the operator $T$, which is atomic with respect to $F^{\prime}$.

Lemma 3.8. Let $T: X_{1} \rightarrow X_{2}$ be atomic with respect to the $\sigma$-homomorphism $F: \tilde{\Sigma}_{1} \rightarrow \tilde{\Sigma}_{2}$, while $\tilde{\Sigma}_{1} \in \Omega\left(\tilde{\Sigma}_{1}^{\prime}\right)$ and $F$ admits an extension to a $\sigma$-homomorphism $F^{\prime}: \tilde{\Sigma}_{1}^{\prime} \rightarrow \tilde{\Sigma}_{2}$. Then the operator $T$ admits the unique extension to the operator $T^{\prime}$ : $X_{1}^{\prime} \rightarrow X_{2}$, which is atomic with respect to $F^{\prime}$. Moreover, the extended operator $T^{\prime}$ is continuous in measure whenever $T$ is continuous in measure.

Proof. Existence. For an arbitrary $v \in X_{1}^{\prime}$ and for every $j \in \mathbb{N}$ there is a $v^{j} \in X_{1}$ such that

$$
\left.v\right|_{\Omega_{1}^{j}}=\left.v^{j}\right|_{\Omega_{1}^{j}}
$$

Now set

$$
\left(T^{\prime} v\right)(x):=\left(T v^{j}\right)(x) \text { for } \mu_{2} \text {-a.e. } x \in F^{\prime}\left(\Omega_{1}^{j}\right) .
$$

Note that the above definition is correct. In fact, although there might exist another $\tilde{v}^{j} \in X_{1}$ such that

$$
\left.v\right|_{\Omega_{1}^{j}}=\left.\tilde{v}^{j}\right|_{\Omega_{1}^{j}}
$$

but clearly then

$$
\left.\tilde{v}^{j}\right|_{\Phi\left(\Omega_{1}^{j}\right)}=\left.v^{j}\right|_{\Phi\left(\Omega_{1}^{j}\right)},
$$

where $\Phi\left(e_{1}\right)$ stands for the minimum (up to a set of $\mu_{1}$-measure zero) set from $\Sigma_{1}$ which contains $e_{1} \in \Sigma_{1}^{\prime}$. Therefore, in view of atomicity of $T$ one has

$$
\left(T \tilde{v}^{j}\right)(x)=\left(T v^{j}\right)(x) \quad \text { for } \mu_{2} \text {-a.e. } x \in F\left(\Phi\left(\Omega_{1}^{j}\right)\right),
$$

and hence

$$
\left(T \tilde{v}^{j}\right)(x)=\left(T v^{j}\right)(x) \text { for } \mu_{2} \text {-a.e. } x \in F^{\prime}\left(\Omega_{1}^{j}\right),
$$

since $F^{\prime}\left(\Omega_{1}^{j}\right) \subset F^{\prime}\left(\Phi\left(\Omega_{1}^{j}\right)\right)=F\left(\Phi\left(\Omega_{1}^{j}\right)\right)$.

It is easy to observe that $T^{\prime}$ is the desired extension (namely, it is atomic with respect to $F^{\prime}$ ). In fact, if $u, v \in X_{1}^{\prime}$ are such that

$$
\left.u\right|_{e_{1}}=\left.v\right|_{e_{1}},
$$

then for each $j \in \mathbb{N}$ there exist $u^{j}, v^{j} \in X_{1}$ such that

$$
\left.u\right|_{\Omega_{1}^{j}}=\left.u^{j}\right|_{\Omega_{1}^{j}},\left.\quad v\right|_{\Omega_{1}^{j}}=\left.v^{j}\right|_{\Omega_{1}^{j}} .
$$


Therefore,

which implies

$$
\left.u^{j}\right|_{\Phi\left(e_{1} \cap \Omega_{1}^{j}\right)}=\left.v^{j}\right|_{\Phi\left(e_{1} \cap \Omega_{1}^{j}\right)},
$$

$$
\left.T u^{j}\right|_{F\left(\Phi\left(e_{1} \cap \Omega_{1}^{j}\right)\right)}=\left.T v^{j}\right|_{F\left(\Phi\left(e_{1} \cap \Omega_{1}^{j}\right)\right)},
$$

in view of atomicity of $T$. Then

$$
\left.T u\right|_{F^{\prime}\left(e_{1} \cap \Omega_{1}^{j}\right)}=\left.T u^{j}\right|_{F^{\prime}\left(e_{1} \cap \Omega_{1}^{j}\right)}=\left.T v^{j}\right|_{F^{\prime}\left(e_{1} \cap \Omega_{1}^{j}\right)}=\left.T v\right|_{F^{\prime}\left(e_{1} \cap \Omega_{1}^{j}\right)},
$$

since $F^{\prime}\left(e_{1} \cap \Omega_{1}^{j}\right) \subset F^{\prime}\left(\Phi\left(e_{1} \cap \Omega_{1}^{j}\right)\right)=F\left(\Phi\left(e_{1} \cap \Omega_{1}^{j}\right)\right)$.

Uniqueness. Let $T_{1}^{\prime}$ and $T_{2}^{\prime}$ both be extensions of $T$ atomic with respect to $F^{\prime}$. Again, for every $v \in X_{1}^{\prime}$ and for every $j \in \mathbb{N}$ there is a $v^{j} \in X_{1}$ such that (3.1) holds. In view of atomicity of $T_{1}^{\prime}$ and $T_{2}^{\prime}$ the restrictions of $T_{1}^{\prime} v$ and $T_{2}^{\prime} v$ to each $F^{\prime}\left(\Omega_{1}^{j}\right)$ coincide with those of $T_{1}^{\prime} v^{j}$ and $T_{2}^{\prime} v^{j}$. But since $T_{1}^{\prime} v^{j}=T_{2}^{\prime} v^{j}=T v^{j}$, then in view of the arbitrariness of $j$ one has $T_{1}^{\prime} v=T_{2}^{\prime} v$.

Continuity. The verification of this property is straightforward. Suppose in fact that $T$ is continuous and let $v_{\nu} \rightarrow v$ in $X_{1}^{\prime}$. For each $j \in \mathbb{N}$, let $v_{\nu}^{j}, v^{j} \in X_{1}$ be such that

$$
\left.v_{\nu}\right|_{\Omega_{1}^{j}}=\left.v_{\nu}^{j}\right|_{\Omega_{1}^{j}},\left.\quad v\right|_{\Omega_{1}^{j}}=\left.v^{j}\right|_{\Omega_{1}^{j}} .
$$

Then one has $v_{\nu}^{j} \rightarrow v^{j}$ in $L^{0}\left(\Phi\left(\Omega_{1}^{j}\right), \Sigma_{1} \cap \Phi\left(\Omega_{1}^{j}\right), \mu_{1}\right)$. Hence $T^{\prime} v_{\nu}=T v_{\nu}^{j} \rightarrow T v_{j}=T^{\prime} v$ in $L^{0}\left(F^{\prime}\left(\Omega_{1}^{j}\right), \Sigma_{2} \cap F\left(\Omega_{1}^{j}\right), \mu_{2}\right)$ and, since the latter holds for all $j \in \mathbb{N}$, one has $T^{\prime} v_{\nu} \rightarrow T v$ in $X_{2}$.

Note that one has uniqueness of the extended operator only when the extension of the respective $\sigma$-homomorphism is fixed. In fact, even a local operator can have several extensions to atomic operators (among which, clearly, there is exactly one local extension), as the following example shows.

Example 3.9. Let $\Omega_{1}=\Omega_{2}=S^{1}$ be a unit circumference, let $\mathcal{X}_{1}=\mathcal{X}_{2}=\mathbb{R}$, and let $\Sigma_{1}^{\prime}=\Sigma_{2}$ be a completion of the Borel $\sigma$-algebra with respect to the one-dimensional Haussdorf measure. Let $g: S^{1} \rightarrow S^{1}$ be the rotation of the circumference by the angle $2 \pi \alpha$. Consider any measurable map $h: S^{1} \rightarrow S^{1}$ such that $(h \circ g)(x)=h(x)$ for a.e. $x \in$ $S^{1}$. Let $\Sigma_{1}:=h^{-1}\left(\Sigma_{1}^{\prime}\right)$. The operator $T: X_{1} \rightarrow X_{2}$ defined by

$$
(T u)(x):=f(x, u(g(x)))
$$

for some Carathéodory function $f: S^{1} \times \mathbb{R} \rightarrow S^{1}$ is clearly local. Fore every $u \in X_{1}$ one has $u \circ g=u$, and hence $(T u)(x)=f(x, u(x))$. Therefore, a continuous local extension $T^{\prime}: X_{1}^{\prime} \rightarrow X_{2}$ of the operator $T$ is clearly given by the formula

$$
\left(T^{\prime} u\right)(x)=f(x, u(x)) .
$$

On the other hand, another continuous atomic extension of this operator to the whole space $X_{1}^{\prime}$ is given by the same formula (3.2). This operator is atomic with respect to $F^{\prime}$ defined by $F^{\prime}\left(\tilde{e}_{1}^{\prime}\right):=\left[g^{-1}\left(e_{1}^{\prime}\right)\right]$, but it is not local. 
We remark that Lemma 3.8 despite being extremely simple has interesting consequences on its own. For instance, one has to mention that its immediate corollary is the following assertion on the extension of local operators proved in $[\mathbf{7}]$.

Corollary 3.10. Let $N: X_{1} \rightarrow X_{2}$ be a local operator, and let $\tilde{\Sigma}_{1} \in \Omega\left(\tilde{\Sigma}_{1}^{\prime}\right)$. Then $N$ admits the unique extension to the local operator $N^{\prime}: X_{1}^{\prime} \rightarrow X_{2}$. Moreover, the extended operator $N^{\prime}$ preserves the continuity of $N$ in measure.

We can now claim the following representation result for continuous-in-measure atomic operators which gives an answer to Problem 3.3.

Theorem 3.11. Let $\left(\Omega_{1}, \Sigma_{1}, \mu_{1}\right)$ and $\left(\Omega_{2}, \Sigma_{2}, \mu_{2}\right)$ be standard measure spaces and let $F: \tilde{\Sigma}_{1} \rightarrow \tilde{\Sigma}_{2}$ be a $\sigma$-homomorphism. Then any continuous operator $T: X_{1} \rightarrow X_{2}$ atomic with respect to $F$ can be represented as

$$
(T u)(x)=f(x, u(g(x))) \quad \text { for } \mu_{2} \text {-a.e. } x \in \Omega_{2}
$$

for some Carathéodory function $f: \Omega_{2} \times \mathcal{X}_{1} \rightarrow \mathcal{X}_{2}$, a measurable function $g: \Omega_{2} \rightarrow \Omega_{1}$ satisfying (2.1) and every $u \in X_{1}$, if and only if $F\left(\tilde{\Sigma}_{1}\right) \in \Omega\left(\tilde{\Sigma}_{2}\right)$.

Proof. Theorem 32.3 of [8] implies the existence of a measurable function $g: \Omega_{2} \rightarrow \Omega_{1}$ satisfying $(2.1)$ and $\left[g^{-1}\left(e_{1}\right)\right]=F\left(\tilde{e}_{1}\right)$ for every $e_{1} \in \Sigma_{1}^{\prime}$. According to the representation Theorem 3.1 from [3] , one has $T=N \circ T_{g}$, where $N$ is a local operator defined over $L^{0}\left(\Omega_{2}, F\left(\tilde{\Sigma}_{1}\right), \mu_{2} ; \mathcal{X}_{1}\right)$. If $F\left(\tilde{\Sigma}_{1}\right) \in \Omega\left(\tilde{\Sigma}_{2}\right)$, then according to Corollary 3.10 the operator $N$ admits a unique continuous local extension to the whole space $L^{0}\left(\Omega_{2}, \Sigma_{2}, \mu_{2} ; \mathcal{X}_{1}\right)$, and hence is representable as

$$
(N v)(x)=f(x, v(x)) \quad \text { for } \mu_{2} \text {-a.e. } x \in \Omega_{2},
$$

where $f: \Omega_{2} \times \mathcal{X}_{1} \rightarrow \mathcal{X}_{2}$ is a Carathéodory function.

Otherwise, if $F\left(\tilde{\Sigma}_{1}\right) \notin \Omega\left(\tilde{\Sigma}_{2}\right)$, then Theorem 7 from $[7]$ asserts the existence of a continuous local operator $N: L^{0}\left(\Omega_{2}, F\left(\tilde{\Sigma}_{1}\right), \mu_{2} ; \mathcal{X}_{1}\right) \rightarrow L^{0}\left(\Omega_{2}, \Sigma_{2}, \mu_{2} ; \mathcal{X}_{2}\right)$, which cannot be represented as a Nemytskiĭ operator generated by a Carathéodory function. Therefore, the operator $T:=N \circ T_{g}$ is atomic with respect to $F$ but cannot be represented as indicated in the statement of the theorem.

\section{The $\Omega$-condition and piecewise-injective functions}

Before studying Problem 3.5, we recall a notion of $\omega$-condition for a function acting between two standard measure spaces. This notion was introduced by Drakhlin in [2] to study a class of shifts in Lebesgue spaces which have 'nice' representation of adjoints.

Definition 4.1. Let $\left(\Omega_{i}, \Sigma_{i}, \mu_{i}\right), i=1,2$, be standard measure spaces. We say that a measurable function $g: \Omega_{2} \rightarrow \Omega_{1}$

(i) is piecewise injective if there exists a disjoint at-most-countable covering of $\Omega_{2}$ by measurable sets $\Omega_{2}=\bigsqcup_{j} \Omega_{2}^{j}$ such that over each $\Omega_{2}^{j}$ the function is injective; 
(ii) satisfies the $\omega$-condition if it is piecewise injective, satisfies (2.1), and the respective inverses $\gamma_{j}: g\left(\Omega_{2}^{j}\right) \rightarrow \Omega_{2}^{j},\left.\gamma_{j} \circ g\right|_{\Omega_{2}^{j}}=$ id satisfy $(2.1)$.

Note that a piecewise-injective function $g: \Omega_{2} \rightarrow \Omega_{1}$ satisfying (2.1) can always be changed on a set of zero $\mu_{2}$-measure in order to satisfy the $\omega$-condition. In fact, consider the measures $\mu_{1}^{j}$ on $\Omega_{1}$ defined by

$$
\mu_{1}^{j}\left(e_{1}\right):=\mu_{2}\left(g^{-1}\left(e_{1}\right) \cap \Omega_{2}^{j}\right)
$$

for every $e_{1} \in \Sigma_{1}^{\prime}$. Since $\mu_{1}^{j}$ are absolutely continuous with respect to $\mu_{1}$, we may consider the respective Radon-Nikodym derivatives $\mathrm{d} \mu_{1}^{j} / \mathrm{d} \mu_{1}$ and set

$$
E_{2}^{j}:=g^{-1}\left(\left\{y: \frac{\mathrm{d} \mu_{1}^{j}}{\mathrm{~d} \mu_{1}}(y)=0\right\}\right) \cap \Omega_{2}^{j} .
$$

Setting now

$$
\tilde{g}(x):= \begin{cases}g(x), & x \in \Omega_{2} \backslash \bigcup_{j} E_{2}^{j}, \\ x_{0}, & x \in \bigcup_{j} E_{2}^{j},\end{cases}
$$

where $x_{0} \in \Omega_{1}$ is arbitrary, and observing that $\mu_{2}\left(E_{2}^{j}\right)=0$, one arrives at the conclusion that $g(x)=\tilde{g}(x)$ for $\mu_{2}$-a.e. $x \in \Omega_{2}$, while $\tilde{g}$ satisfies the $\omega$-condition. Therefore, we will just identify the piecewise-injective functions satisfying (2.1) with the functions satisfying the $\omega$-condition.

We find it convenient to also provide the following simple and rather folkloric lemma.

Lemma 4.2. Let $g: \Omega_{2} \rightarrow \Omega_{1}$ satisfy the $\omega$-condition. Then there is a Borel set $\Omega_{2}^{\prime} \subset \Omega_{2}$ with $\mu_{2}\left(\Omega_{2} \backslash \Omega_{2}^{\prime}\right)=0$, such that $\Omega_{2}^{\prime}=\bigsqcup_{j} \Omega_{2}^{\prime j}$, where all $\Omega_{2}^{\prime j}$ are Borel sets, while the restrictions $\left.g\right|_{\Omega_{2}^{\prime j}}$ are Borel measurable, injective and possess Borel-measurable inverses $\gamma_{j}$ which satisfy $(2.1)$.

Proof. Let $\Omega_{2}=\bigsqcup_{j} \Omega_{2}^{j}$, where $\Omega_{2}^{j}$ are injectivity sets for $g: \Omega_{2} \rightarrow \Omega_{2}$, as indicated in Definition 4.1. It is enough to show that for each $j$ there is a Borel set $\Omega_{2}^{\prime j} \subset \Omega_{2}^{j}$ with $\mu_{2}\left(\Omega_{2}^{j} \backslash \Omega_{2}^{\prime j}\right)=0$, while the restriction of $g$ to $\Omega_{2}^{\prime j}$ is Borel measurable. Borel measurability of inverses $\gamma_{j}$ will follow then from the Kuratowski-Suslin Theorem (Theorem 8.3.7 of [1]), and putting $\Omega_{2}^{\prime}:=\bigsqcup_{j} \Omega_{2}^{\prime j}$ we will conclude the proof of the lemma.

To prove this claim, pick up a countable set of generators $\mathcal{G}=\left\{G_{\nu}\right\}$ of $\mathcal{B}_{1}$, i.e. $\sigma(\mathcal{G})=$ $\mathcal{B}_{1}$, where $\sigma(\mathcal{G})$ stands for the minimum $\sigma$-algebra generated by $\mathcal{G}$, and $\mathcal{B}_{i}$ stands for the Borel $\sigma$-algebra of $\Omega_{i}, i=1,2$. For each $\nu \in \mathbb{N}$ one has

$$
g^{-1}\left(G_{\nu}\right) \cap \Omega_{2}^{j}=B_{j, \nu}^{\prime} \cup C_{j, \nu}^{\prime},
$$

where $B_{j, \nu}^{\prime} \in \mathcal{B}_{2}$ and $C_{j, \nu}^{\prime} \subset C_{j, \nu} \in \mathcal{B}_{2}, \mu_{2}\left(C_{j, \nu}\right)=0$. Let $D_{j} \in \mathcal{B}_{2}$ be such that $\mu_{2}\left(\Omega_{2}^{j} \backslash D_{j}\right)=0$ and set

$$
\Omega_{2}^{\prime j}:=D_{j} \backslash \bigcup_{\nu} C_{j, \nu}
$$


Clearly, $\Omega_{2}^{\prime j}$ is a Borel set, while $\mu_{2}\left(\Omega_{2}^{j} \backslash \Omega_{2}^{\prime j}\right)=0$. One now has

$$
g^{-1}\left(G_{\nu}\right) \cap \Omega_{2}^{\prime j}=B_{j, \nu}^{\prime} \cap \Omega_{2}^{\prime j} \in \mathcal{B}_{2},
$$

for every $\nu \in \mathbb{N}$, which proves that the restriction of $g$ to $\Omega_{2}^{\prime j}$ is Borel measurable, and hence concludes the proof.

The above lemma has a very easy but rather significant consequence formulated in the proposition below.

Proposition 4.3. Let $\left(\Omega_{i}, \Sigma_{i}, \mu_{i}\right), i=1,2$, be standard measure spaces, while $g$ : $\Omega_{2} \rightarrow \Omega_{1}$ satisfy the $\omega$-condition. Then $g^{-1}\left(\tilde{\Sigma}_{1}\right) \in \Omega\left(\tilde{\Sigma}_{2}\right)$.

Proof. Let $\Omega_{2}^{\prime}=\bigsqcup_{j} \Omega_{2}^{\prime j}$ be as in the statement of Lemma 4.2. We will show that $g^{-1}\left(\Sigma_{1}\right) \cap \Omega_{2}^{\prime j} / \Sigma_{2}^{0}=\Sigma_{2} \cap \Omega_{2}^{\prime j} / \Sigma_{2}^{0}$ for all $j$, where $\Sigma_{2}^{0}$ stands for the $\sigma$-ideal of $\mu_{2}$-nullsets. The only non-trivial inclusion to be proven is

$$
\Sigma_{2} \cap \Omega_{2}^{\prime j} / \Sigma_{2}^{0} \subset g^{-1}\left(\Sigma_{1}\right) \cap \Omega_{2}^{\prime j} / \Sigma_{2}^{0} .
$$

To show the latter, consider an arbitrary $\tilde{e}_{2} \in \Sigma_{2} \cap \Omega_{2}^{\prime j} / \Sigma_{2}^{0}$ and let $e_{2} \subset \tilde{e}_{2}$ be such a Borel set that $\left[e_{2}\right]=\tilde{e}_{2}$. Clearly, $e_{2} \cap \Omega_{2}^{\prime j}$ is a Borel set and since $g$ is injective over $\Omega_{2}^{\prime j}$ and has a Borel-measurable inverse, then

$$
e_{2} \cap \Omega_{2}^{\prime j}=g^{-1}\left(g\left(e_{2} \cap \Omega_{2}^{\prime j}\right)\right) \cap \Omega_{2}^{\prime j} .
$$

Minding that $\left[e_{2} \cap \Omega_{2}^{\prime j}\right]=\tilde{e}_{2}$ and that $g\left(e_{2} \cap \Omega_{2}^{\prime j}\right)=\gamma_{j}^{-1}\left(e_{2}\right) \in \Sigma_{1}$ is a Borel set suffices to conclude the proof.

The above proposition asserts, roughly speaking, that a function between standard measure spaces satisfying the $\omega$-condition generates a $\sigma$-algebra satisfying the $\Omega$-condition. Below we will prove that in a sense a reverse statement is valid, namely, that every $\sigma$-subalgebra of a standard measure space which satisfies the $\Omega$-condition is generated by some function satisfying the $\omega$-condition.

\section{Extensions of $\sigma$-homomorphisms}

In this section we will study Problem 3.5 and we will see that it is in fact quite closely related to Problems 3.3 and 3.4. The answer to this problem is given by the following proposition.

Proposition 5.1. Let $\tilde{\Sigma}_{1} \in \Omega\left(\tilde{\Sigma}_{1}^{\prime}\right)$. Then every $\sigma$-homomorphism $F: \tilde{\Sigma}_{1} \rightarrow \tilde{\Sigma}_{2}$ admits an extension to a $\sigma$-homomorphism $F^{\prime}: \tilde{\Sigma}_{1}^{\prime} \rightarrow \tilde{\Sigma}_{2}$.

Proof. According to the assumptions there is an at-most-countable covering $\Omega_{1}=$ $\bigsqcup_{j} \Omega_{1}^{j}, \Omega_{1}^{j} \in \Sigma_{1}^{\prime}$, such that for each $j \in \mathbb{N}$ one has $\Sigma_{1} \cap \Omega_{1}^{j} / \Sigma_{1}^{0}=\Sigma_{1}^{\prime} \cap \Omega_{1}^{j} / \Sigma_{1}^{0}$. Observe that for each $e_{1}^{\prime} \in \tilde{\Sigma}_{1}^{\prime}$ the minimum $\Sigma_{1}$-measurable set containing $e_{1}^{\prime}$ is defined uniquely up to a set of measure zero, and thus denote the equivalence class of the latter by 
$\Phi\left(e_{1}^{\prime}\right)$. Note that $\Phi: \Sigma_{1}^{\prime} \cap \Omega_{1}^{j} / \Sigma_{1}^{0} \rightarrow \tilde{\Sigma}_{1}$ is a $\sigma$-homomorphism. In fact, the only nontrivial property to verify is $\Phi\left(e_{1}^{\prime}\right) \cap \Phi\left(e_{1}^{\prime \prime}\right)=\emptyset$ whenever $e_{1}^{\prime} \cap e_{1}^{\prime \prime}=\emptyset$. Suppose this is not true, i.e. there exist such disjoint $e_{1}^{\prime}, e_{1}^{\prime \prime} \in \Sigma_{1}^{\prime} \cap \Omega_{1}^{j} / \Sigma_{1}^{0}$ that the set $T:=\Phi\left(e_{1}^{\prime}\right) \cap \Phi\left(e_{1}^{\prime \prime}\right)$ has positive measure. In view of the $\Omega$-condition, one has $\Phi\left(e_{1}^{\prime}\right) \cap \Omega_{1}^{j}=e_{1}^{\prime}$ and $\Phi\left(e_{1}^{\prime \prime}\right) \cap \Omega_{1}^{j}=e_{1}^{\prime \prime}$, and hence, since $e_{1}^{\prime}$ and $e_{1}^{\prime \prime}$ are disjoint, $T \cap\left(e_{1}^{\prime} \cup e_{1}^{\prime \prime}\right)=\emptyset$. It is easy to observe now that the set $\Phi\left(e_{1}^{\prime}\right) \backslash T \in \Sigma_{1}$ contains $e_{1}^{\prime}$ but has measure strictly less than that of $\Phi\left(e_{1}^{\prime}\right)$, which contradicts the definition of $\Phi$.

If $e_{1}^{\prime j} \in \Sigma_{1}^{\prime} \cap \Omega_{1}^{j}$, define

$$
F^{\prime j}\left(e_{1}^{\prime j}\right):=F\left(e_{1}^{j}\right) \cap \Omega_{2}^{j}, \quad \text { where } e_{1}^{j}:=\Phi\left(e_{1}^{\prime j}\right) \text { and } \Omega_{2}^{j}:=F\left(\Phi\left(\Omega_{1}^{j}\right)\right) .
$$

Then, clearly, $F^{\prime j}: \Sigma_{1}^{\prime} \cap \Omega_{1}^{j} / \Sigma_{1}^{0} \rightarrow \Sigma_{2} \cap \Omega_{2}^{j} / \Sigma_{2}^{0}$ is a $\sigma$-homomorphism. The desired extension is now given by

$$
F^{\prime}\left(e_{1}^{\prime}\right):=\bigsqcup_{j} F^{\prime j}\left(e_{1}^{\prime} \cap \Omega_{1}^{j}\right)
$$

for every $e_{1}^{\prime} \in \tilde{\Sigma}_{1}^{\prime}$.

We can claim more, namely that the above proposition in a certain sense completely characterizes the $\Omega$-condition. For this purpose consider first the following example.

Example 5.2. Let $\Omega_{1}=(0,1) \times(0,1), \Sigma_{1}^{\prime}$ being the usual Lebesgue $\sigma$-algebra of $\Omega_{1}$ and $\mu_{1}$ being the two-dimensional Lebesgue measure, while $\Omega_{2}=(0,1), \Sigma_{2}$ is the Lebesgue $\sigma$-algebra of $\Omega_{2}$ and $\mu_{2}$ is the one-dimensional Lebesgue measure. Let $\Sigma_{1} \subset$ $\Sigma_{1}^{\prime}$ stand for the $\sigma$-algebra of the sets $e_{2} \times(0,1)$, where $e_{2} \in \Sigma_{2}$, and define the $\sigma$ homomorphism $F: \tilde{\Sigma}_{1} \rightarrow \tilde{\Sigma}_{2}$ by setting

$$
F\left(\left[e_{2} \times(0,1)\right]\right):=\tilde{e}_{2} .
$$

Clearly, $\tilde{\Sigma}_{1} \notin \Omega\left(\tilde{\Sigma}_{1}^{\prime}\right)$. Moreover, in this case $F$ cannot be extended to the whole $\tilde{\Sigma}_{1}^{\prime}$. In fact, if it were possible, then by Theorem 32.3 of [8] there would exist a measurable function $g: \Omega_{2} \rightarrow \Omega_{1}$ satisfying $(2.1)$ and $\left[g^{-1}\left(e_{1}\right)\right]=F\left(\tilde{e}_{1}\right)$ for every $e_{1} \in \Sigma_{1}^{\prime}$, that is,

$$
\left[g^{-1}\left(e_{2} \times(0,1)\right)\right]=\tilde{e}_{2} .
$$

Since $g=(\phi, \psi)$, where $\phi, \psi: \Omega_{2} \rightarrow \Omega_{2}$ are the components of the function $g$, then the above relationship implies

$$
\left[\phi^{-1}\left(e_{2}\right)\right]=\tilde{e}_{2}
$$

for all $\tilde{e}_{2} \in \tilde{\Sigma}_{2}$, and therefore $\phi(z)=z$ a.e. on $\Omega_{2}$. In other words, $g(z)=(z, \psi(z))$ for some measurable function $\psi:(0,1) \rightarrow(0,1)$. The set $G:=\operatorname{graph} \phi$ then has Lebesgue measure zero in $\Omega_{1}$, but $g^{-1}(G)=\Omega_{2}$, hence contradicting (2.1).

We now extend the above example to the more general situation.

Proposition 5.3. Let $\left(\Omega_{1}, \Sigma_{1}^{\prime}, \mu_{1}\right)$ be a standard measure space and $\tilde{\Sigma}_{1} \notin \Omega\left(\tilde{\Sigma}_{1}^{\prime}\right)$. Then there exists a $\sigma$-homomorphism $F: \tilde{\Sigma}_{1} \rightarrow \tilde{\Sigma}_{2}$ which admits no extension to a $\sigma$ homomorphism $F^{\prime}: \tilde{\Sigma}_{1}^{\prime} \rightarrow \tilde{\Sigma}_{2}$. 
Proof. We take $\left(\Omega_{2}, \Sigma_{2}, \mu_{2}\right)=\left(\Omega_{1}, \Sigma_{1}^{\prime}, \mu_{1}\right)$. There are two possibilities: either $\tilde{\Sigma}_{1}=g^{-1}\left(\tilde{\Sigma}_{1}^{\prime}\right)$ for some measurable function $g: \Omega_{2} \rightarrow \Omega_{1}$ satisfying (2.1), or else $\tilde{\Sigma}_{1}$ is not generated by any function. In the latter case it is enough to take the identity $\sigma$-homomorphism for $F$. In fact, if it were extendable to the whole $\tilde{\Sigma}_{1}^{\prime}$, then by Theorem 32.3 in [8] one would have $F\left(\tilde{e}_{1}\right)=\left[g^{-1}\left(e_{1}\right)\right]$ for some measurable $g: \Omega_{2} \rightarrow \Omega_{1}$ satisfying (2.1), and, hence, $\tilde{\Sigma}_{1}=g^{-1}\left(\tilde{\Sigma}_{1}^{\prime}\right)$, contradicting the assumption. We concentrate therefore on the former case, namely, when $\tilde{\Sigma}_{1}=g^{-1}\left(\tilde{\Sigma}_{1}^{\prime}\right)$. Let us remark that the function $g$ cannot be piecewise injective, since otherwise $\Sigma_{1}$ would clearly satisfy the $\Omega$ condition according to Proposition 4.3. Restricting, if necessary, to a set of full measure in $\Omega_{2}$, we may suppose that $g$ is Borel measurable. Let $E_{2}^{\prime} \subset \Omega_{2}, \mu_{2}\left(E_{2}^{\prime}\right)>0$ be such that the restriction of $g$ to $E_{2}^{\prime}$ is anti-injective, that is, for every $e_{2} \subset E_{2}^{\prime}, e_{2} \in \Sigma_{2}$ one has $\mu_{2}\left(e_{2}\right)=0$ whenever $\left.g\right|_{e_{2}}$ is injective. Suppose also without loss of generality that $\mu_{2}\left(E_{2}^{\prime}\right)=1$ (otherwise just re-norm the measure). By Proposition 2.2 from [6], which is a consequence of the Maharam Theorem on homogeneous measure algebras, there then exist a Borel set $E_{2} \subset E_{2}^{\prime}, \mu_{2}\left(E_{2}\right)=\mu_{2}\left(E_{2}^{\prime}\right)=1$, a compact metric space $M$, a nonatomic Borel probability measure $\mathbb{P}$ on $M$, and a Borel-measurable map $\tau: E_{2} \rightarrow M$ such that the following conditions hold:

(a) the map $g \times \tau: E_{2} \rightarrow \Omega_{1} \times M$ defined by

$$
(g \times \tau)(x):=(g(x), \tau(x))
$$

is invertible;

(b) the image measure $\mu^{\prime}$ over $\Omega_{1} \times M$ defined by

$$
\mu^{\prime}(B):=\mu_{2}\left((g \times \tau)^{-1}(B)\right)
$$

for all Borel sets $B \subset \Omega \times M$ satisfies

$$
\mu^{\prime}=\mu_{g} \otimes \mathbb{P}, \quad \text { where } \mu_{g}\left(e_{1}\right):=\mu_{2}\left(g^{-1}\left(e_{1}\right)\right) .
$$

For the moment we identify $g$ with its restriction to $E_{2}$, so that $g: E_{2} \rightarrow \Omega_{1}$. It is easy to see that

$$
g(x)=p_{\Omega_{1}}((g \times \tau)(x)),
$$

where $p_{\Omega_{1}}: \Omega_{1} \times M \rightarrow \Omega_{1}$ is the projection map. Now set $F\left(\left[g^{-1}\left(e_{1}\right)\right]\right):=\tilde{e}_{1}$ for $e_{1} \in \Sigma_{1}^{\prime}$. Defined in this way, $F: \tilde{\Sigma}_{1} \cap E_{2} \rightarrow \tilde{\Sigma}_{2}$ is a $\sigma$-homomorphism in view of (5.1). Denote by $\hat{\Sigma}_{1}^{\prime}$ the completion with respect to $\mu^{\prime}$ of the Borel $\sigma$-algebra of $\Omega_{1} \times M$, and by $\hat{\Sigma}_{1}$ the $\sigma$-algebra of subsets of $\Omega_{1} \times M$ of the form $e_{1} \times M, e_{1} \in \Sigma_{1}$. We note that $F$ induces a $\sigma$-homomorphism

$$
\hat{F}: \tilde{\hat{\Sigma}}_{1} \rightarrow \tilde{\Sigma}_{2}
$$

according to the formula

$$
\hat{F}\left(\hat{e}_{1}\right):=F\left((g \times \tau)^{-1}\left(\hat{e}_{1}\right)\right)
$$


In fact, $\hat{F}: p_{\Omega_{1}}^{-1}\left(e_{1}\right) \mapsto e_{1}$. Suppose that $F$ can be extended to $F^{\prime}$ defined over the whole $\tilde{\Sigma}_{1}^{\prime} \cap E_{2}$. Then $\hat{F}$ would be extendable to an

$$
\hat{F}^{\prime}: \tilde{\hat{\Sigma}}_{1}^{\prime} \rightarrow \tilde{\Sigma}_{2}
$$

according to the formula

$$
\hat{F}\left(\hat{e}_{1}^{\prime}\right):=F\left((g \times \tau)^{-1}\left(\hat{e}_{1}^{\prime}\right)\right),
$$

and the reasoning of Example 5.2 shows that this is impossible.

\section{Characterization of the $\Omega$-condition}

Proposition 5.1 has an interesting consequence which extends the famous theorem of von Neumann on the representation of $\sigma$-homomorphisms $($ see $[\mathbf{5}, \mathbf{1 0}]$ or Theorem 32.3 in $[8])$.

Corollary 6.1. Let $\tilde{\Sigma}_{1} \in \Omega\left(\tilde{\Sigma}_{1}^{\prime}\right)$, while $\left(\Omega_{1}, \Sigma_{1}^{\prime}, \mu_{1}\right)$ is a standard measure space. Then every $\sigma$-homomorphism $F: \tilde{\Sigma}_{1} \rightarrow \tilde{\Sigma}_{2}$ is generated by a measurable map $g: \Omega_{2} \rightarrow \Omega_{1}$ satisfying (2.1), namely,

$$
\left[g^{-1}\left(e_{1}\right)\right]=F\left(\tilde{e}_{1}\right)
$$

for all $e_{1} \in \Sigma_{1}$. In particular, there is a measurable map $h: \Omega_{1} \rightarrow \Omega_{1}$ satisfying (2.1) such that $\tilde{\Sigma}_{1}=h^{-1}\left(\tilde{\Sigma}_{1}^{\prime}\right)$.

Proof. Every $\sigma$-homomorphism $F: \tilde{\Sigma}_{1} \rightarrow \tilde{\Sigma}_{2}$ can be extended by Proposition 5.1 to a $\sigma$-homomorphism $F^{\prime}: \tilde{\Sigma}_{1}^{\prime} \rightarrow \tilde{\Sigma}_{2}$. It is enough to apply the representation Theorem 32.3 of $[8]$ to the latter to conclude the proof of the first assertion. To prove the existence of the map $h$ which generates the measure algebra $\tilde{\Sigma}_{1}^{\prime}$, one applies the representation result to the identity $\sigma$-homomorphism.

Now we can completely characterize the $\Omega$-condition on $\sigma$-subalgebras in standard measure spaces. In fact, the theorem below gives the desired result on characterization of the $\Omega$-condition on $\sigma$-algebras in terms of the existence of a generating function satisfying the $\omega$-condition.

Theorem 6.2. Let $\left(\Omega, \Sigma^{\prime}, \mu\right)$ be a standard measure space with non-atomic measure and $\Sigma \subset \Sigma^{\prime}$. Then $\tilde{\Sigma} \in \Omega\left(\tilde{\Sigma}^{\prime}\right)$ if and only if there is a function $h: \Omega \rightarrow \Omega$ satisfying the $\omega$-condition, such that $\tilde{\Sigma}=h^{-1}\left(\tilde{\Sigma}^{\prime}\right)$. Moreover, every measurable function $h: \Omega \rightarrow \Omega$ satisfying (2.1) such that $\tilde{\Sigma}=h^{-1}\left(\tilde{\Sigma}^{\prime}\right)$ also satisfies the $\omega$-condition.

Proof. If $h: \Omega \rightarrow \Omega$ satisfies the $\omega$-condition, then according to Proposition 4.3 one has $h^{-1}\left(\tilde{\Sigma}^{\prime}\right) \in \Omega\left(\tilde{\Sigma}^{\prime}\right)$. We prove therefore the reverse statement. Namely, suppose that $\tilde{\Sigma} \in \Omega\left(\tilde{\Sigma}^{\prime}\right)$. Then according to Corollary 6.1 there is a measurable $h: \Omega \rightarrow \Omega$ satisfying (2.1) such that $\tilde{\Sigma}=h^{-1}\left(\tilde{\Sigma}^{\prime}\right)$. On the other hand, the $\Omega$-condition implies the existence of an at-most-countable cover $\Omega=\bigsqcup_{j} \Omega^{j}$ consisting of sets $\Omega_{j} \in \Sigma^{\prime}$ of positive measure such that

$$
h^{-1}\left(\Sigma^{\prime}\right) \cap \Omega^{j} / \Sigma^{0}=\Sigma^{\prime} \cap \Omega^{j} / \Sigma^{0} \text { for all } j \in \mathbb{N},
$$


where $\Sigma^{0} \subset \Sigma^{\prime}$ stands for the $\sigma$-ideal of $\mu$-nullsets. In other words, for all $e \in \Sigma^{\prime}, e \subset \Omega^{j}$ (with $j \in \mathbb{N}$ fixed) there is an $e^{\prime} \in \Sigma^{\prime}$ such that $h^{-1}\left(e^{\prime}\right) \cap \Omega^{j}=e$ modulo a $\mu$-nullset. In order to prove that $h$ satisfies the $\omega$-condition, it suffices to show that so does its restriction to each $\Omega^{j}$. Suppose that the latter is not true for some $j \in \mathbb{N}$. In view of Proposition 3.1 from [9], there is then a set $E \subset \Omega^{j}$ with $\mu(E)>0$ and a non-atomic $\sigma$-algebra $\hat{\Sigma}$ of subsets of $E$ such that $h^{-1}\left(\Sigma^{\prime}\right) \cap E$ and $\hat{\Sigma}$ are independent, that is, for all $e_{1} \in h^{-1}\left(\Sigma^{\prime}\right) \cap E$ and $e_{2} \in \hat{\Sigma}$ one has

$$
\mu_{E}\left(e_{1} \cap e_{2}\right)=\mu_{E}\left(e_{1}\right) \mu_{E}\left(e_{2}\right), \quad \text { where } \mu_{E}(e):=\mu(e) / \mu(E) .
$$

Taking an arbitrary $e \in \hat{\Sigma}$, one has for the respective $e^{\prime}$ satisfying $h^{-1}\left(e^{\prime}\right) \cap E=e$ the relationship

$$
\mu_{E}(e)=\mu_{E}\left(e \cap\left(h^{-1}\left(e^{\prime}\right) \cap E\right)\right)=\mu_{E}(e) \mu_{E}\left(h^{-1}\left(e^{\prime}\right) \cap E\right)=\mu_{E}(e)^{2},
$$

which implies that either $\mu_{E}(e)=0$ or $\mu_{E}(e)=1$, contradicting the non-atomicity of $\hat{\Sigma}$.

Clearly, the requirement of non-atomicity of the measure $\mu$ is essential in the above theorem. In fact, consider the following example.

Example 6.3. Let $\Omega=(0,1), \Sigma^{\prime}$ be the Lebesgue $\sigma$-algebra of $\Omega$ and let $\mu$ be the point measure defined by

$$
\mu(e):= \begin{cases}1, & x_{0} \in e, \\ 0, & x_{0} \notin e,\end{cases}
$$

for a given $x_{0} \in \Omega$. Then clearly $\tilde{\Sigma}^{\prime}=\{\tilde{\emptyset}, \tilde{\Omega}\}$. We consider $\Sigma:=\Sigma^{\prime}$. Then the function $g: \Omega \rightarrow \Omega$ defined by $g(x):=x_{0}$ for all $x \in \Omega$ generates $\tilde{\Sigma}=\tilde{\Sigma}^{\prime}$ but is not piecewise injective.

\section{References}

1. D. L. Cohn, Measure theory (Birkhäuser, 1980).

2. M. E. Drakhlin, On one linear functional equation, in Functional differential equations, pp. 91-111 (Perm Polytechnical Institute, Perm, 1985), in Russian.

3. M. Drakhlin, A. Ponosov and E. Stepanov, On some classes of operators determined by the structure of their memory, Proc. Edinb. Math. Soc. 45 (2002), 467-490.

4. D. H. Fremlin, Measure algebras, in Handbook of Boolean algebras (ed. J. D. Monk and R. Bonnet), vol. 3 (North-Holland, 1989).

5. P. R. Halmos and J. von Neumann, Operator methods in classical mechanics, II, Ann. Math. 43 (1942), 332-350.

6. N. J. KALton, Isomorphisms between $L_{p}$-function spaces when $p<1$, J. Funct. Analysis 42(3) (1981), 299-337.

7. Yu. NePomnyash'IKh AND A. Ponosov, Local operators in some subspaces of the space $L_{0}$, Izv. VUZ Mat. 6 (1999), 50-64, in Russian.

8. R. Sikorski, Boolean algebras (Springer, 1960).

9. E. Stepanov AND A. Zdorovtsev, Relaxation of some nonlocal integral functionals in weak topology of Lebesgue space, J. Convex Analysis 8 (2001), 447-469.

10. J. VOn NEumAnn, Einige Sätze über die meßbaren Abbildungen, Ann. Math. 33 (1932), $574-586$, in German. 